\title{
Narratologische Probleme des faktualen Erzählens
}

\section{Monika Fludernik}

Wie schon in der Einleitung erörtert, ist das faktuale Erzählen ein Stiefkind der narratologischen Forschung geblieben und erst kürzlich ins Rampenlicht der Erzähltheorie getreten. In diesem Beitrag möchte ich kurz mögliche Unterschiede zwischen faktualem und fiktionalem Erzählen Revue passieren lassen und dann anhand eines Beispiels genauer die Problemlage auf der Diskursebene diskutieren.

Wenn man faktuales und fiktionales Erzählen gegenüberstellt, kann man sich mehrerer Modelle der Fiktionalität oder des Fiktionalen, der Fiktion, bedienen. Referentialität stellt für viele Forscher ein grundlegendes Kriterium dar (faktuale oder nicht-fiktionale Texte sind welche, in denen auf real vorhandene oder auf existierende Entitäten und Räume Bezug genommen wird, während die Personen und Schauplätze von fiktionalen Erzählungen nicht existent, sondern erfunden sind). Innerhalb des GRK 1767 wird jedoch die kommunikative These vertreten, nach der die (oft nicht nachprüfbare) Frage der Erfundenheit nachrangig ist und fiktionale oder faktuale Texte als solche produziert und auch als solche rezipiert werden. Danach liegt dieses Kriterium für eine Klassifikation im Kontext begründet (Gattung, Publikationsmedium, Autorintention, Lesereinstellung; vgl. dazu Zipfel 2001: 279 ff.). Nicht nur, dass fiktionale Texte, z. B. Romane, häufig auf Realreferenten Bezug nehmen (Churchill, Obama oder London, Istanbul); literarische Texte und Filme haben zudem den Anspruch, Wahres und Signifikantes über die Welt und den Menschen auszusagen, etwa in Anlehnung an Aristoteles, der zwischen der Historie, die sagen muss, wie es wirklich gewesen, und der поınбıs, die sagen kann, wie es (idealerweise) hätte sein können, unterscheidet (Aristoteles 1994: IX). Bareis (2008) vertritt sogar die Ansicht, dass es fiktionale Texte geben kann, die ausschließlich aus faktischen Elementen bestehen, weshalb er Fiktivität nicht als Definiens der Fiktion erachtet.

Die Position des GRK 1767 inkludiert, stimmt aber nicht völlig überein mit derjenigen von Kendall Walton (1990) (den Bareis proponiert), wonach die Fiktion ausschließlich auf der Rezipienten-Ebene zu verorten wäre - Objekte (u. a. Texte) könnten zwar für ihre Rezeption als Fiktion produziert werden, aber Leser oder Rezipienten von Kunst würden Gegenstände und Texte im Rahmen eines vorsätzlichen „make believe“ in Fiktion transformieren. Dieser rein relativistischen These: Fiktion ist, was Rezipienten als solche betrachten oder was sie praktisch als solche ,anwenden', steht entgegen, dass Fiktionalität unter manchen Umständen sozial als Skandalon aufgefasst werden kann und Tabubrüche zu initiieren scheint, wobei nicht nur die Tatsache der Fiktivität, also des Erfundenseins, Anstoß zu erregen vermag, sondern auch das Was und Wie der Darstellung des Erfundenen (Franzen 2014). Skandalöse Texte oder Kunstwerke (vgl. Steiner 1995) werden ge- 
rade deshalb als anstößig empfunden, weil sie als faktisch rezipiert werden, ohne das ,willing suspension of disbelief', das für die Kunst nötig sei.

Das GRK 1767 hält an der prinzipiellen Unterscheidung von faktualen und fiktionalen Texten fest und richtet sich so gegen die Thesen des Panfiktionalismus. ${ }^{1}$ Uns interessiert vor allem, wie faktuale Texte im Gegensatz zu fiktionalen ,funktionieren' und wie in bestimmten Gattungen so wie der des Dokudramas (vgl. den Beitrag von Barbara Korte in diesem Band) die Unterscheidung absichtlich verwischt wird oder wie Werke mit der Transgression zwischen den beiden Bereichen ästhetisch spielen (wie in Hildesheimers Marbot). Es ist also nach unserer Auffassung der Erzählakt faktual bzw. fiktional; die dargestellten Welten können real oder fiktiv sein.

In allen diesen Definitionen und Überlegungen zur Fiktionalität, die ich der Kürze halber hier in stark vereinfachter Form resümieren musste, geht es um Textmodelle, in denen berichtet wird, also beschreibende Aussagen über Entitäten getroffen werden, was wohl auch mit dem Fokus auf die Existenz fiktiver bzw. realer Individuen oder Räume zusammenhängt. In ihrem einschlägigen Buch Wirklichkeitserzäblungen (2009) haben Christian Klein und Matías Martínez jedoch darauf hingewiesen, dass es neben deskriptiven auch normative und visionäre Erzählungen gibt, deren Bezug zur Wirklichkeit, sprechakttheoretisch gesprochen, nicht rein konstativ ist. So wollen normative Erzählungen Entwürfe vermitteln, wie etwas sein soll, und zielen auf eine didaktische und moralische Beeinflussung des Rezipienten, während visionäre Texte zwar fiktiv sind, weil das, was sie beschreiben, nicht existiert, aber per visionärem Wahrheitsanspruch behaupten, dass diese Ereignisse sein werden, also durchaus faktual (in der Zukunft) wären. Daraus lässt sich als erster wichtiger Unterschied zwischen faktualen und fiktionalen Texten festhalten, dass faktuale Erzählungen eine viel offensichtlichere und zentralere Instrumentalisierung der Narration zu ihrer Praxis gemacht haben.

Ein zweites Kriterium der Unterscheidung zwischen faktualen und fiktionalen Erzählungen liegt in der wohlbekannten Distinktion zwischen Autor und Erzähler, die für das literarische Feld konstitutiv ist (vgl. Genette 1993: 25, Cohn 1999: 32 ff.). Die Erschaffung einer Erzählerfigur durch den namensverschiedenen Autor kennzeichnet eine Erzählung als fiktional. Dabei dient die Erzählerfigur oft der Verschleierung der Autorschaft, aber oft auch der Selbst-Distanzierung, z. B. in der Lyrik, wo das lyrische Ich (im Englischen speaker of the poem) sich in eine Rolle versetzt und diese quasi fiktiv ausprobiert. Des Weiteren kann die Erzählerfiktion als Rabmungsstrategie fungieren. Autofiktion (vgl. den Beitrag von Jutta Weiser in diesem Band) ist gerade deshalb ein so interessantes Phänomen, weil die Erfahrungsgleichheit zwischen Autor und Figur und ihre häufige Namenskoinzidenz, gepaart mit der Gattungsbezeichnung „Roman“, genau diese traditionelle Grenzmarkierung zwischen Faktualität und Fiktionalität unterminiert.

1 Zum Panfiktionalismus siehe Ryan (1997, 2005) sowie Konrad (2014). 
Als drittes Kriterium der Unterscheidung fungiert fast kanonisch die Betonung innerer Vorgänge bzw. die Bewusstseinsdarstellung von Protagonisten. Obwohl die Historiographie vor dem 20. Jahrhundert sowie moderne Experimente wie diejenigen Simon Schamas (1991) dieses Kriterium ignorieren bzw. unterlaufen haben, gilt doch cum grano salis, dass ausfübrliche Bewusstseinsdarstellung dem Roman, also der Fiktion, vorbehalten bleibt. Mit diesem Befund in Zusammenhang stehen auch theoretische Modelle der Erzählforschung, insbesondere die Frage nach der Narrativität, also dessen, was eine Erzählung ausmacht, wie sie definiert werden kann. Klassische Modelle der Erzähltheorie (Stanzel, Genette, Schmidt 2005) betonen die Ereignisfolge, den Plot, als konstitutives Element der Erzählung, während ich selbst (und siehe auch Ryan 2004) experientiality, also „Erlebnisqualität“ oder „Erfahrungshaftigkeit“, vorgeschlagen habe (Fludernik 1996, 2013). In der Gegenüberstellung von faktualen und fiktionalen Erzählungen scheint die traditionelle Definition die adäquatere zu sein: Beinahe alle Gattungen von schriftlichen faktualen Erzählungen basieren auf einer Handlungsfolge, während Erlebnisqualität in der Form von Bewusstseinsdarstellung eher eine untergeordnete Rolle spielt. Nur im mündlichen Erzählen, welches ich als Basis für meine Theorien in Towards a, Natural ${ }^{c}$ Narratology verwendete, spielt Erlebnishaftigkeit eine fast ebenso zentrale Rolle wie im fiktionalen Erzählen.

Dieser Befund ist besonders spannend. Das mündliche Alltagserzählen enthält in mehrerer Hinsicht fiktionale (fiktive) Elemente: erfundene Dialoge und Gedanken der Protagonisten, starke chronologische Konturierungen mit Rückblenden und Antizipationen, performanzbezogene Privilegierung von affektvoller Darstellung (z. B. in der Form von Übertreibung, Verwendung von Klischees, unspezifische und ungefähre Referenz) mit einhergehender Nachreichung von wahrheitsgetreuer Präzision. Andererseits weist mündliches Erzählen in Alltagsgesprächen auch eine starke Betonung des Erzählkontextes auf, der einen klaren Realitätsbezug markiert. Die Erzählung wird eingesetzt, um eigene Leistungen mit Nachdruck zu belegen, um face zu manipulieren, um sich in der Konkurrenz mit Mitsprechern zu behaupten. Die erzählten Geschichten haben so trotz ihrer unterhaltenden Funktion auch dezidiert argumentative und konstatierende Intentionen, welche an Exempla in mittelalterlichen Predigten erinnern. (Vgl. auch Anekdoten in historischen Darstellungen oder Parabeln.) Diese unmittelbare Inanspruchnahme der Geschichte durch den Erzähler unterscheidet auch das mündliche Erzählen mit seinen vielen fiktionalen Elementen von der Fiktion, wie wir sie in Kurzgeschichten, Romanen oder Filmen antreffen. Vielfach sind mündliche Erzählungen auf der hypodiegetischen Erzählebene angesiedelt, also gerahmte Narrationen.

Wie Cohn (1999), Löschnigg (1999) und Fludernik (2001) ausgeführt haben, ist Unzuverlässigkeit ein weiteres Unterscheidungskriterium von Fiktionalität. Nur in fiktionalen Texten kann es einen unzuverlässigen Erzähler in der Art geben, wie dies typisch für fiktionale Erzählungen ist. Es stehen zwar alle Erzähler unter dem generellen Verdacht der Unzuverlässigkeit, aber in fiktionalen Erzäh- 
lungen wird diese Unzuverlässigkeit als intendierter literarischer Effekt rezipiert und nicht als Zufall oder Rahmenbedingung interpretiert (Fludernik 2005). Der Effekt der unzuverlässigen Erzählung liegt genau darin, daß der Leser die Unzuverlässigkeit erkennt und zwischen den Zeilen des Berichts dieses Ich-Erzählers liest und so eine Gegengeschichte konstruiert.

Weitere Eigenschaften, die faktuale und fiktionale Texte unterscheiden, sind gradueller Natur. Nach dem Modell von Fludernik (2000) - siehe Diagramm 1 kann man argumentieren, dass der Diskurs des faktualen Erzählens stärker berichtbezogen ist. Ein konstativer Sprechakt liegt in vielen Gattungen vor; die Erzählungen führen aus, was sich wo ereignet hat, wobei instruktive Texte ihre Handlungsanleitungen mit reichem deskriptiven Beiwerk rahmen. Kochbücher oder Reiseführer sind insofern sehr interessante faktuale Erzähltextsorten, da sie Handlungen darstellen, die zum Zeitpunkt des Schreibens fiktiv sind, jedoch vom Leser beim Kochen oder bei der Stadtbesichtigung realisiert werden. Sie ähneln deshalb den visionären Texten, die Klein und Martínez in ihren Wirklichkeitserzählungen (2009) aufführen.

Wie im Diagramm dargelegt, enthalten die Textsorten höchster Ebene (macrogenres, vgl. Hempfer 1973) auf der Ebene des Textes grundsätzlich alle Diskurstypen (Berichten, also report, Beschreiben, Argumentieren, Kommentieren, etc.); individuelle Texte oder Gattungen hingegen mögen bestimmte Diskurstypen prioritär einsetzen, können aber nicht auf einen Diskurstyp reduziert werden. Ergo wird Erzählung nicht nur durch Berichtsätze gekennzeichnet; wissenschaftliche Prosa hat nicht nur Sätze von Argumentation, sondern enthält Beschreibungen, Berichte und Kommentare, usw. Nach diesem Modell kann man daher den Widerspruch zwischen den verschiedenen Definitionen von Narrativität dahingehend abschwächen, dass man die faktuale Erzählung in viel stärkerem Maße als Bericht-lastig charakterisiert und so grundsätzlich nur den Anteil der Handlungsfolgen referierenden Textteile variiert.

Ein weiterer Unterschied zwischen fiktionalen und faktualen Erzählungen mag in der Signifikanz von Immersion (Ryan 2001) für die fiktionale Erzählung liegen. Immersion, oder das Sich-Vertiefen in die erzählte Welt, wird oft auch als Korrelat der Emotivität von Fiktion gesehen. Man könnte vielleicht argumentieren, dass der emotionale Effekt von fiktionaler Literatur möglicherweise bei faktualen Texten nicht nur durch Distanzierung, sondern auch durch Evaluation abgegolten wird - fiktionale Texte rufen eher Gefühle des Sich-Hineinversenkens hervor, während bei faktualen Texten die kritische Analyse und das anwendungsbezogene Verständnis der Erzählung emotiv wirken. Ich lehne mich hier an Weinrichs Unterscheidung von Besprechen und Erzählen (1985) an, transferiere diese Einsicht jedoch in die Unterschiedung von faktualen und fiktionalen Texten.

Ferner ist zu konstatieren, dass in faktualen Textsorten das Erzählen überhaupt häufig einen geringen Prozentsatz ausmacht, da meist das Argumentieren vorherrscht. Dies ist besonders in der Geschichtsschreibung und in wissenschaftli- 
Figure 2.

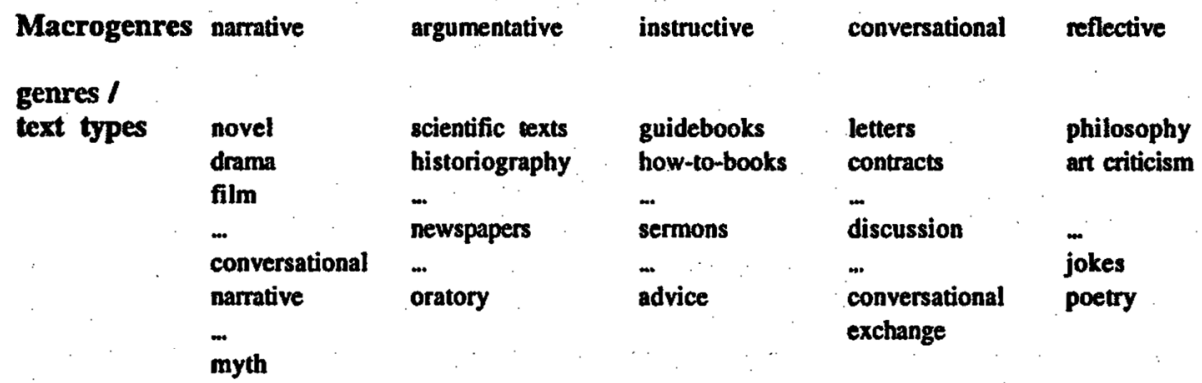

\begin{tabular}{|c|c|c|c|c|c|}
\hline nodes & $\begin{array}{l}\begin{array}{l}\text { report } \\
\text { sequence }\end{array} \\
\text { orientational } \\
\text { passages } \\
\text { (=description) }\end{array}$ & $\begin{array}{l}\text { expositional } \\
\text { sentences } \\
\text { argumentative } \\
\text { passages } \\
\text { (commentary) }\end{array}$ & $\begin{array}{l}\text { directives } \\
\text { exhortations }\end{array}$ & $\begin{array}{l}\text { address } \\
\text { dialogue } \\
\text { phatic } \\
\text { sequences }\end{array}$ & $\begin{array}{l}\text { metalinguistic } \\
\text { statements } \\
\text { gnomic } \\
\text { language } \\
\text { word play }\end{array}$ \\
\hline
\end{tabular}

Diagramm 1: Drei Ebenen der gattungskonstituierenden Textsorten (aus: Fludernik 2000)

chen Arbeiten der Fall (außer dort, wo Examenskandidaten Inhaltsangaben statt Analysen präsentieren). Häufig finden sich Erzählungen überhaupt nur als Inset, als Illustration, Erklärung und Veranschaulichung, da in der Funktion von Parabeln und Exempeln. Solche Einsprengsel in den argumentativen Text können sowohl (auto)biographisch und emotional sowie betont erfahrungsbezogen sein wie auch implizit metaphorisch, wenn sie parabelhaft eine Situation schildern, die dann als Analogon zur behandelten Fragestellung fungiert. Eine Diskussion über das Recht des Stärkeren kann etwa mit der Fabel vom Wolf und dem Lämmlein illustriert werden, um die implizite Lehre zu unterstreichen, dass sich Recht gegen blanke Gewalt nicht durchzusetzen vermag. Man könnte bei solchen Kontexten sogar von einer gerabmten Fiktionalität sprechen: die Fiktionalität der Fabel wird zu pragmatischen Zwecken der Argumentation eingesetzt. Hier kann man dann nur bedingt von faktualen Erzäblungen reden.

Ein weiterer wichtiger Aspekt faktualen Erzählens bezieht sich darauf, dass faktuales Erzählen immer rezeptionsbezogen ist: Für faktuales Erzählen gilt wie für alle Kommunikation das Message-Modell - der Autor will dem Leser etwas mitteilen. Beim fiktionalen Erzählen hingegen bereitet das Message-Modell einige Proble$m e,^{2}$ da sich die fiktionale Erzählung besonders seit dem Roman schon ob ihrer Komplexität einer einfachen Kommunikationsstruktur entzieht. Romane fordern

2 Zur Kritik an einer rein kommunikationsorientierten Narratologie siehe u. a. Fludernik (1993) sowie Köppe/Stühring (2011). Dezidierte kommunikationsorientierte Modelle der Erzähltheorie sind u. a. Nünning (1989), Sell (2000) und Hogan (2013). 
uns eher auf, uns in eine fiktionale Welt hineinzuversetzen. Ihre Aussagen werden meist nicht explizit - und dann nur im Rahmen der Äußerungen des Erzählers - dargelegt, sondern müssen, und das oft auf widersprüchliche Weise, von den Lesern erschlossen werden.

Ein letztes wichtiges Unterscheidungsmerkmal zwischen fiktionalen und faktualen Erzählungen ist die Existenz von kollektiven und deanthropomorphen Handlungsträgern in faktualen Erzählungen. Obwohl es mittlerweile immer mehr literarische Wir-Erzählungen gibt, ${ }^{3}$ ist die Form der Wir-Erzählung in faktualen Erzählungen prominent und weit verbreitet, und das nicht nur in mündlichen Erzählungen über Kriegserlebnisse oder Familienurlaube. Noch klarer ist die Spezifizität des faktualen Erzählens, wenn man auf Erzählungen stößt, die (wie häufig in der Geschichtsschreibung) Staaten oder Institutionen als Handlungsträger inszenieren. Diese spielen auch in bürokratischen und administrativen Textsorten eine gewichtige Rolle (Pensionäre, Arbeitslose, Handwerker, Jugendliche, usw.), wobei die narrativen Anteile in diesen Textsorten meist gering sind, aber Berichte über Entwicklungen in der Vergangenheit durchaus vorkommen.

\section{Ein Fallbeispiel: Beschreibung}

Im Folgenden möchte ich anhand einer Fallstudie eine ganz andere Herangehensweise erproben, als die, welche in den schon genannten Arbeiten zur Unterscheidung von fiktionalen und faktualen Erzählungen bzw. Texten verfolgt wurden. Diese Forschungen hatten alle zum Ziel, Fiktionalität global Faktualität gegenüber zu stellen, sei es für Texte im Allgemeinen oder für Erzählungen im Besonderen. Dabei ist jedoch zu vermerken, dass sowohl auf der Seite fiktionaler Erzählungen (um mal nur Erzähltexte zu betrachten) wie seitens faktualer Erzählungen jeweils eine Vielzahl verschiedener Gattungen existieren, in denen daher wohl auch möglicherweise ganz verschiedene Techniken zur Anwendung kommen, wenn es um die Bestimmung der Fiktionalität bzw. Faktualität dieser Texte geht. Dabei denke ich vor allem an Fiktionssignale im Hempferschen Sinn (Hempfer 1990). Wenn man z. B. nur historische Schriften analysiert, kann man verschiedene Untergattungen der Historiographie unterscheiden - z. B. nicht nur Annalen, Chroniken und Historiographie im engeren Sinne, sondern auch Herrscherbiographien, Geschichten einer Epoche, Nationalgeschichten, Geschichten einer Institution, Mentalitätsgeschichten, usw. Es liegt auf der Hand, dass die Oberflächenstruktur dieser Texte (ihrer narrative Diskurse) sehr verschieden sind und auch Aspekte der Fiktionalisierung in manchen dieser Gattungen sehr selten, in andern eher häufiger anzutreffen sind. Über diese Gattungsfragen hinaus stellt sich jedoch auch die Frage, wie bestimmte Elemente des narrativen Diskurses in fiktionalen bzw. faktualen Text(sort)en fungieren und ob hier Unterschiede bestehen.

3 Zur Wir-Erzählung vgl. u. a. Margolin (1996, 2000), Marcus (2008), Richardson (2011). 
Mein Beispiel ist die Beschreibung. Man könnte jedoch auch die chronologische Struktur, die Funktionen der Erzähler-/Autorkommentare, u. a. m. untersuchen. Meine Wahl ist auf die Beschreibung gefallen, weil sie in faktualen Texten eine große Rolle spielt (besonders in Handlungsanleitungen wie Reiseführern) und weil sie in der Erzählforschung bis auf wenige Ausnahmen als Forschungsgegenstand sträflich vernachlässigt wurde - die Ausnahmen sind neben Hamon (1981, 1982) und Bal (1982) auch Wolf/Bernhart (2007).

Als Erstes muss man sich darüber einigen, was man überhaupt unter dem Begriff Beschreibung versteht. Es ist eine häufige Erfahrung, dass Leser und auch Literaturwissenschaftler meinen, dass eine Beschreibung intuitiv als solche erkannt werden kann. Leider stellt sich die Definitionsfrage bei näherer Betrachtung als komplexer heraus. ${ }^{4}$ Nach Sichtung der Literatur gibt es meiner Ansicht nach folgende Definitionsmöglichkeiten:

(1) Beschreibungen attribuieren Eigenschaften an beschriebene Objekte und Personen, deren Existenz so behauptet wird. Also ist ein großer Raum groß, ein schwarzhaariger Mann besitzt schwarze Haare und ein geschmackvolles Fauteuil erfüllt bestimmte ästhetische Kriterien. Dieses letzte Beispiel zeigt bereits ein Problem der Definition auf, nämlich die Subjektivität der Attribute und ihres Wertekanons und die Notwendigkeit, Garanten für diese impliziten Behauptungen zu finden.

(2) Neben dieser logischen Definition hat die Erzählforschung (siehe besonders Chatman 1978, 1990) die Beschreibung als jene Diskursstrategie gesetzt, welche im Gegensatz zu Ereignissen für die Einführung von existents zuständig ist. Diese inkludieren Räume (Schauplätze) und Personen (Chatman 1978: 96 ff.). Diese sehr gängige Definition setzt eine prinzipielle Dichotomie zwischen Handlungsbericht (narrative report) und Beschreibung (description) voraus, also zwischen Kinetik und Statik, was wiederum reichlich problematisch ist, da in der Forschung mehrfach auf die Ununterscheidbarkeit von Beschreibung und Handlungsbericht hingewiesen wurde (Mosher 1991). Ein Satz wie Er hob lächelnd seine Hand und runzelte seine Stirn, so dass sie sich in dicke Falten legte beschreibt sowohl Handlungen wie auch visuelle Eindrücke (lächelndes Gesicht, Falten), die normalerweise als ,beschreibend' klassifiziert würden. Eine ähnliche Verbrämung von Handlungen und Eigenschaftszuschreibungen findet sich in Kochbüchern und Reiseführern, die häufig als deskriptive Gattungen betrachtet werden. Auch schulische Leistungsbeschreibungen, wie sie in der Linguistik untersucht werden (Kotthoff 2012), bestehen aus Sätzen wie Er sagt kaum etwas und schreibt immer vom Nachbarn ab. Hier wird die Beschreibung der Leistung durch Auflistung von typischen Verhaltensweisen realisiert.

4 Siehe die Darstellung in der Einleitung des Sonderhefts von Style 48.4 (2014/15), das von Suzanne Keen und Monika Fludernik herausgegeben wurde. Besonders interessante Arbeiten zur Beschreibung sind neben Bal (1981) und Mosher (1991) auch Lodge (1977) sowie Sternberg $(1974,1981)$. 
Eine Konsequenz von Chatmans Modell, die sich auch schon bei Genette findet, ist die Tatsache, dass, während der Text beschreibt, die Handlung stillsteht und nichts passiert. Die Beschreibung, wie der Erzählerkommentar, ist also etwas, das in den Pausen der Erzählung stattfindet. Diese Betrachtungsweise ergibt sich insbesondere bei Analyse des viktorianischen Romans oder der Werke Balzacs und Flauberts, die in den Aufsätzen von Bal und Hamon sowie in den Studien von Wolf/Bernhart und Bonheim vorrangig figurieren.

(3) Eine dritte, an (2) angelehnte Möglichkeit ist es, die Beschreibung temporal im Verhältnis zur Handlung zu charakterisieren: Auf der Plot-Ebene folgen die Ereignisse einander; in Beschreibungen, selbst wenn es sich um Prozesse handelt, werden Hintergrundinformation dargeboten; sie dienen als orientation im Sinne von Labov/Waletzkys Modell mündlichen Erzählens (1968). Eine Erklärung, wie die Papierproduktion funktioniert, enthält viele Hinweise auf Prozesse, die ablaufen, und auf die Handlungen der Arbeiterinnen in der Fabrik (ich denke an Melvilles Geschichte „The Paradise of Bachelors and the Tartarus of Maids“, 1855). Diese Handlungen und Prozesse sind jedoch nicht Teil des Plots, und sie sind rekurrent.

(4) Eine weitere Möglichkeit, Beschreibung zu definieren, ist, alles was Sinneseindrücke vermittelt als Beschreibung zu behandeln. Dies bietet sich für fiktionale Erzählungen besonders an, wo Beschreibungen meist diejenigen Momente im Handlungsgeschehen markieren, an denen Protagonisten in einen Raum treten und sehen bzw. hören, was dort existiert oder vorfällt. Auch wenn der Leser vom Erzähler an einen neuen Schauplatz gebracht oder mit einer neuen Romanfigur bekanntgemacht wird, finden sich häufig Beschreibungen en bloque. Diese Definition hat jedoch ebenfalls den Nachteil, die Vermischung von Erzählen und Beschreibung nicht adäquat zu reflektieren, und sie mag für faktuale Texte nicht so überzeugen.

(5) Werner Wolf (2007: 16) hat drei verschiedene Typen von Beschreibungsfunktionen vorgestellt, die den Funktionen von (a) Identifikation (er nennt das die „referential function“), (b) Revisualisierung (,representational and experiential function“) und (c) Faktenvermittlung über ein Objekt („pseudo-objectivizing and interpretative function") entsprechen. Beschreibungen erlauben es im ersteren Fall, ein Objekt oder eine Person wiederzuerkennen, zu identifizieren. Im zweiten Fall wird das Objekt imaginär vom Leser visualisiert und vermittelt einen Wahrnehmungseindruck. Die dritte Kategorie ist quasi definitorisch, sie soll den Gegenstand charakterisieren, so wie dies die Wörterbuchdefinition des Pferdes in der Eröffnungsszene des zweiten Kapitels von Dickens' Hard Times tut: „Quadruped. Graminivorous. Forty teeth, namely twenty-four grinders, four eye-teeth, and twelve incisive. Sheds coat in the spring; in marshy countries, sheds hoofs, too. Hoofs hard, but requiring to be shod with iron. Age known by marks in mouth"(Dickens 1854: 6). 
Diese drei Kategorien erlauben es zudem, abstrakte Beschreibungen (Eine Kaffeemaschine besteht aus ...), die eher in faktualen Textsorten vorkommen, von narrativen Beschreibungen zu trennen, welche implizit fokalisieren, also eigentlich aussagen, dass das Objekt X zum Zeitpunkt Y so aussah. Von dort ist es dann nicht weit zur paysage mentalisé, zu Romanszenen, in denen die Protagonistin aus dem Fenster sieht und ihre Gefühle und Stimmung auf die Landschaft überträgt. Wo in Wolfs Schema auktoriale Beschreibungen angesiedelt wären, wurde mir jedoch nicht so klar.

Neben diesen Definitions- bzw. Kategorisierungsansätzen, die größtenteils auf dem Was der Beschreibung basieren, stellt sich zusätzlich die Frage nach dem Wie oder der Form der Beschreibung. Wie Nünning (2007) in seinem Schema klar ausführt, kann Beschreibung in Blockform oder intermittierenden Sätzen oder gar nur Phrasen, Adjektiven und Adverbien im Text aufscheinen. Sie kann nur zur Vorstellung von Schauplatz und Personen am Textbeginn vorkommen oder an verschiedenen Stellen des Texts und mit variabler Intensität bzw. Rekurrenz.

Die Linguistik hat sich wiederum ganz anders mit dem Thema Beschreibung auseinandergesetzt, wenn man Arbeiten wie die von Klotz (2013) ausnimmt, der sich auch sehr intensiv mit literarischer Beschreibung in Prosa und Lyrik befaßt. So interessiert sich die Linguistik besonders für Referenz- und Deixis-Fragen. Schwitalla (2012) unterscheidet etwa drei Arten der Referenz: (a) absolute (z. B. im Norden); (b) deiktische (gegenüber); und (c) intrinsische (vor dem Haus, wo vor diejenige Seite des Hauses meint, an der die Eingangstür liegt, also auf der ,kanonischen' Vorderfront des Hauses). Aus diesen Standardunterscheidungen der Linguistik abgeleitet hatten Linde/Labov (1975) und danach Taylor/Tversky (1992, 1996) für Raumbeschreibungen zwei statische und eine dynamische Perspektive unterschieden, nämlich:

(1) die Vogelperspektive (survey perspective), in der von oben vertikal wie auf ein Reißbrett auf den Raum gesehen wird und die absolute Referenz vorherrscht;

(2) die sogenannte Beobachtungsperspektive (gaze perspective, dt. Blickwanderung [Ullmer-Ehrich 1979: 65] oder Blickperspektive), in der horizontal Raum beschrieben wird unter Einsatz deiktischer Referenzelemente; und

(3) die route perspective, welche einen dynamischen, immersiven und oft fiktiven oder imaginären Rundgang durchführt, mit wechselnder deiktischer Raumreferenz.

Während bei (2) also, nehmen wir Abbildung 1 als Beispiel, ein Beschreiber sagen könnte: Ich stehe vor dem Hotel, rechts gegenüber ist ein Zeitschriftenladen und links gegenüber das Theater. Auf meiner Linken befindet sich die Apotheke und rechts eine Telefonzelle, würde im Falle von (3) ein Tourist durch die Stadt geleitet, dem man z.B. sagt: Um zur Bank zu kommen, gehen Sie vom Hotel auf der Lane Street nach rechts und biegen nach links in die Queen Avenue ein. Sie gehen die Queen Avenue entlang und pas- 
sieren auf der rechten Seite das Polizeirevier und das Spital. Zu Ibrer Linken ist ein großer Parkplatz, der sich bis zur Palm Street erstreckt. Sie gehen um den Parkplatz herum, biegen also nach links in die Palm Street ein und gehen noch vor, vorbei am Supermarkt zu Ibrer Linken, bis Sie auf der rechten Straßenseite die Bank gleich neben dem koreanischen Restaurant sehen. Auch hier sind alle Raum-Ausdrücke (lokaldeiktische Raumausdrücke und Präpositionen) auf die Origo (Bühler 1934) des imaginären Touristen bezogen; dieser ist jedoch im Raum unterwegs, wodurch sich sein deiktisches Zentrum ständig verlagert. Nach Ullmer-Ehrich (die sich auf Wunderlich bezieht), handelt es sich um sekundäre Deixis, da die Deiktika nur mittelbar die Origo etablieren und nicht direkt auf einen Gesprächsakt referieren (1979:61).

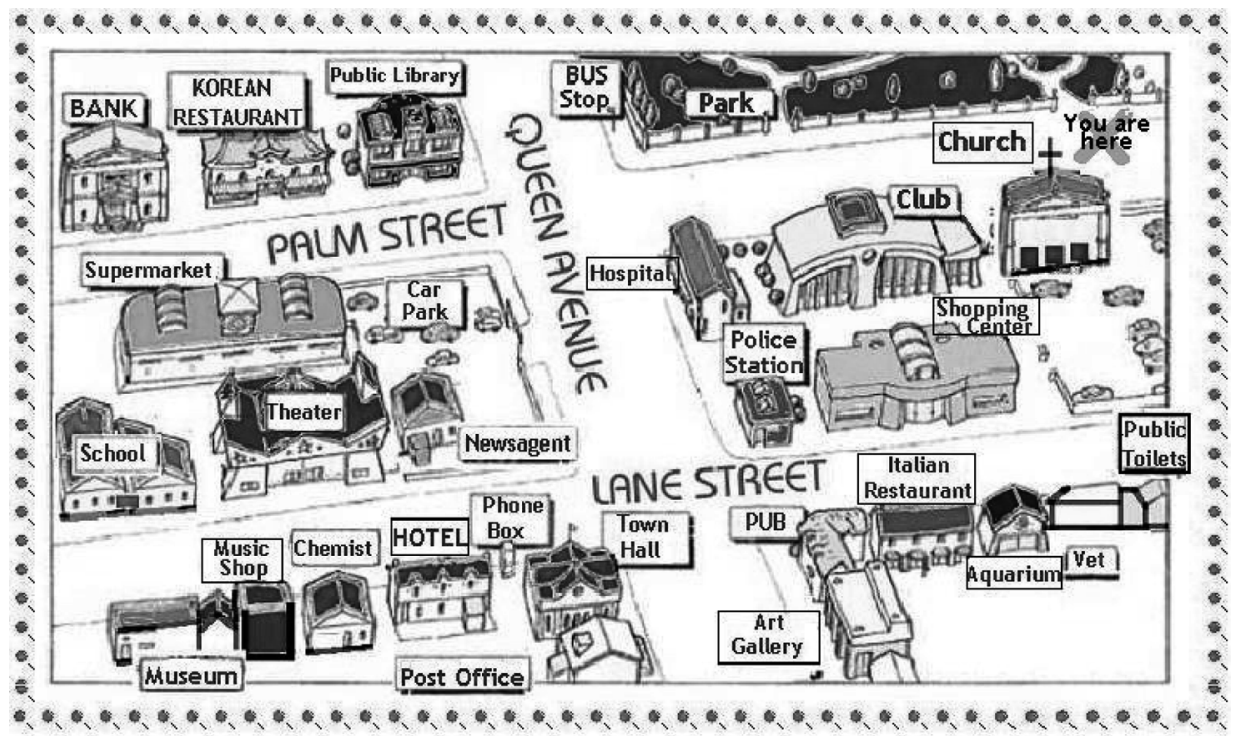

Abbildung 1: Straßenansicht (English Channel. „Practice giving directions“) <http://tx.englishch.com/teacher/jocelyn/level-b/practice-giving-directions/ $>$ (Stand: 26. Nov. 14)

Taylor/Tversky $(1992,1996)$, aber auch Tversky et al. (1999), haben neben Wegbeschreibungen (vgl. auch Klein 1979) auch Wohnungsbeschreibungen analysiert und festgestellt, dass im mündlichen Gespräch die route perspective dominiert, aber teilweise auch mit der gaze perspective kombiniert wird, während absolute Deixis in nichtwissenschaftlichen Texten fast gar nicht zu finden ist. Besonders bei Wegbeschreibungen spielt zudem die kognitive Stütze der sogenannten landmarks eine große Rolle. Wie in meinem fingierten Text gezeigt, ist Orientierung im Raum stark an markante Stationen gebunden, die dem Wegsuchenden ermöglichen, abzuschätzen, ob er/sie sich noch auf dem richtigen Weg befindet.

Will man nun dieses Modell auf literarische Beschreibungen von Räumen übertragen, ergeben sich interessante Einsichten. Ich rekurriere hier auf einen in der Forschung weniger rezipierten Teil von Franz Karl Stanzels Erzäbltheorie, in dem er sich mit der Frage der Perspektivierung von Beschreibung im Roman aus- 
einandersetzt. Seine These ist, dass vor dem späten 19. Jahrhundert, wo sich die personale Erzählsituation durchzusetzen begann und vermehrt die Dinge durch die Wahrnehmung eines Protagonisten (statt des auktorialen Erzählers) geschildert wurden, ein aperspektivischer Beschreibungsmodus vorläge, während in der personalen Erzählsituation ein perspektivischer Bescheibungsmodus vorherrsche.

Sehen wir uns Stanzels Beispiel für eine typische aperspektivische Raumbeschreibung an: ${ }^{5}$

His lordship was at home, and the two visitors were shown through the accustomed hall into the well-known room where the good old bishop used to sit. The furniture had been bought at a valuation, and every chair and table, every bookshelf against the wall, and every square in the carpet was as well known to each of them as their own bedrooms. Nevertheless they at once felt that they were strangers there. The furniture was for the most part the same, yet the place had been metamorphosed. A new sofa had been introduced, a horrid chintz affair, most unprelatical and almost irreligious; such a sofa as never yet stood in the study of any decent High Church clergyman of the Church of England. The old curtains had also given way. They had, to be sure, become dingy, and that which had been originally a rich and goodly ruby had degenerated into a reddish brown. Mr. Harding, however, thought the old reddish-brown much preferable to the gaudy buff-coloured trumpery moreen which Mrs. Proudie had deemed good enough for her husband's own room in the provincial city of Barchester.

Our friends found Dr. Proudie sitting on the old bishop's chair, looking very nice in his new apron; they found, too, Mr. Slope standing on the hearth-rug, persuasive and eager, just as the archdeacon used to stand; but on the sofa they also found Mrs. Proudie, an innovation for which a precedent might in vain be sought in all the annals of the Barchester bishopric! (Barchester Towers v; Trollope 1990: 33-4; zit. Stanzel 2008: 162-63)

Diese Textstelle gibt einerseits den Eindruck wieder, den die zwei Kleriker empfangen, wie sie das Zimmer betreten. Sie registrieren zunächst das Bekannte und ereifern sich danach an den Änderungen, die alle auf die Einmischung von Mrs. Proudie zurückgehen, welche als Eindringen in die Männerdomäne der anglikanischen Kirche betrachtet werden kann. Die Schandflecke, die Mrs. Proudie im Raum installiert hat, beginnen bei den Vorhängen und dem Sofa und enden mit ihrer physischen Präsenz im Büro des Bischofs. Der Grund, warum (wie Manfred Jahn 1999 ausführt) es schwer fällt, diese Darstellung als aperspektivisch zu sehen, liegt darin begründet, dass die Beschreibung fokalisiert ist - sie gibt den Blick und die Einstellung von Dr. Grantly und Mr. Harding auf die Szene wieder. Da Fokalisierung für den perspektivischen Modus bei Stanzel definitorisch zentral ist, heißt das auch, dass die Kategorisierung zunächst unlogisch scheint. Was Stanzel jedoch meint, ist, dass wir nicht eine Zeichnung des Raumes anfertigen können. Es ist nicht möglich, sich zu entscheiden, was links oder rechts im Raum zu verorten wäre. Die Beschreibung zählt auffällige Rauminhalte auf - ist nach der Eigenschaft der saliency strukturiert - und nicht nach der Reproduzierbarkeit der dargestellten (imaginären)

5 Die Hervorhebungen in diesem und den folgenden Blockzitaten erfolgen durch die Autorin. 
Wirklichkeit. Stanzels Perspektive betrachtet also visuelle Reproduzierbarkeit bzw. Orientierung im Raum; und Fokalisierung kann, muss aber nicht solche Perspektivierung nach sich ziehen.

Wenn man sich von diesem Befund zurück zum linguistischen Modell wendet, sieht man, dass Trollope eine gaze perspective einsetzt; allerdings obne deiktische Ausdrücke, da nur ein relationaler Ausdruck vorhanden ist (against the wall). Wie Ullmer-Ehrich (1979) ausführt, wird von Informanten, die ihre Zimmer beschreiben sollen, meist eine Inventarliste geliefert; erst auf Rückfrage erhält man genauere Lokalisierungen einzelner Möbelstücke im Raum. In der Geschichte des frühen Romans gibt es in der Tat nicht nur kaum Beschreibungen, die ein Nachzeichnen erlauben würden; es gibt sogar fast überhaupt kaum Beschreibungen von Innenräumen und ihrem Mobiliar, da bis ins 17. Jahrhundert Räume eher leer waren und Tische, Bänke und Stühle an die Wände gestellt wurden, um bei Bedarf in die Mitte des Zimmers gerückt zu werden (Wall 2006, Brown 2008). Eine gemütliche Stube oder ein Wohnzimmer mit Inventar, das fest an einen Ort im Raum gebunden war, gab es erst ab dem 18. Jahrhundert und wurde erst im 19. Jahrhundert generell üblich für die Mittel- und Oberschicht. Wie Wall darlegt, fokussierten Betrachter von Innenräumen auf die Qualität von Vorhängen und Stoffen oder Kunstwerken, nicht auf die Anordnung derselben im Raum (Wall 2006).

Typische Passagen im Roman vor 1800 zeigen, wie wenig Raumbeschreibung existierte. So ist der folgende Ausschnitt aus David Simple (1744) ganz typisch:

The next Day, David went to see her again, and as my Lady -- was gone to make a Visit, he met with Cynthia alone: He found her dissolved in Tears, and in such an Agony, that she was hardly able to speak to him [...]. (David Simple II, viii; Fielding 1994: 220-21)

Cynthia befindet sich in einem Zimmer, höchstwahrscheinlich dem parlour, aber dies wie auch die genaueren Raumverhältnisse bleiben ausgespart. Ähnliches lässt sich bei Defoe und Henry Fielding beobachten sowie bei Nashe, Behn oder Gascoigne. Selbst eine Hausbeschreibung, wie sie Pepys für Audley-End gibt, fokussiert auf auffällige Prachtobjekte oder architektonische features und nicht auf eine nachvollziehbare Führung durch die Räumlichkeiten:

[...] Took coach to Audley-End, and did go all over the house and garden; and mighty merry we were. The house indeed do appear very fine, but not so fine as it hath heretofore to me; particularly the ceilings are not so good as I always took them to be, being nothing so well wrought as my Lord Chancellor's are; and though the figure of the house without be very extraordinary good, yet the stayre-case is exceeding poor; and a great many pictures, and not one good one in the house but one of Harry the Eighth done by Holben [sic]; and not one good suit of hangings in all the house, but all most ancient things, such as I would not give the hanging-up of in my house; and the other furniture, beds and other things, accordingly. Only the gallery is good, and above all things the cellars, where we went down and drank of much good liquor. And indeed the cellars are fine: and here my wife and I did sing to my great content. (The Concise Pepys; Pepys 1997: 606) 
Nur selten gibt es eine annähernd perspektivische Darstellung, die einem einen Eindruck vermittelt, wie der Grundriss des Hauses oder die Hausführung ausgesehen haben mögen. Ein solches Beispiel ist in Defoes Schilderung von Wilton House zu finden, die als eine frühe Inszenierung einer tour perspective angesehen werden kann.

You ascend the great staircase at the upper end of the hall, which is very large; at the foot of the staircase you have a Bacchus as large as life, done in fine Peloponnesian marble, carrying a young Bacchus on his arm, the young one eating grapes, and letting you see by his countenance that he is pleased with the taste of them. [...] One ought to stop every two steps of this staircase, as we go up, to contemplate the vast variety of pictures that cover the walls, and of some of the best masters in Europe; and yet this is but an introduction to what is beyond them.

When you are entered the apartments, such variety seizes you every way that you scarce know to which hand to turn yourself. First on one side you see several rooms filled with paintings as before, all so curious, and the variety such, that it is with reluctance that you can turn from them; while looking another way you are called off by a vast collection of busts and pieces of the greatest antiquity of the kind, both Greek and Romans; among these there is one of the Roman emperor Marcus Aurelius in bassorelievo. I never saw anything like what appears here, except in the chamber of rarities at Munich in Bavaria.

Passing these, you come into several large rooms, as if contrived for the reception of the beautiful guests that take them up; one of these is near seventy feet long, and the ceiling twenty-six feet high, with another adjoining of the same height and breadth, but not so long. Those together might be called the Great Gallery of Wilton, and might vie for paintings with the Gallery of Luxembourg, in the Faubourg of Paris.

These two rooms are filled with the family pieces of the house of Herbert, most of them by Lilly or Vandyke [...].

After we have seen this fine range of beauties - for such, indeed, they are - far from being at an end of your surprise, you have three or four rooms still upon the same floor, filled with wonders as before. Nothing can be finer than the pictures themselves, nothing more surprising than the number of them. At length you descend the back stairs, which are in themselves large, though not like the other. (A Tour through the Whole Island of Great Britain; Defoe 1986: 198-99)

Hier wird der Besucher und somit der Leser (man beachte das häufig eingesetzte you) in einer imaginären Wanderung (der Ausdruck von Ullmer-Ehrich 1979) die Treppe hinaufgeleitet, dann durch die einzelnen Räume geführt und zuletzt über eine Hintertreppe wieder ins Parterre zurückgebracht. Die Beschreibung der einzelnen Räume an sich ist jedoch aperspektivisch im Stanzelschen Sinn, da man keinen Eindruck von der Anordnung der Gegenstände innerhalb ihrer Standorte hat.

Der genauen Verortung von Objekten der Wahrnehmung in der Beschreibung steht auch die Funktion der pittoresken Vermittlung visueller Eindrücke entgegen, welche in Romanen vorherrscht. So ist die folgende Passage aus Little Dorrit ganz typisch dafür, dass zwar sehr viel Information übermittelt wird, jedoch die Imagination des Lesers diese so verarbeitet, dass er/sie kein genaues Bild von der Szene anfertigen könnte: 
There was a string of people already straggling in, whom it was not difficult to identify as the nondescript messengers, go-betweens, and errand-bearers of the place. Some of them had been lounging in the rain until the gate should open; others, who had timed their arrival with greater nicety, were coming up now, and passing in with damp whitey-brown paper bags from the grocers, loaves of bread, lumps of butter, eggs, milk, and the like. The shabbiness of these attendants upon shabbiness, the poverty of these insolvent waiters upon insolvency, was a sight to see. Such threadbare coats and trousers, such fusty gowns and shawls, such squashed hats and bonnets, such boots and shoes, such umbrellas and walking-sticks, never were seen in Rag Fair. All of them wore the cast-off clothes of other men and women, were made up of patches and pieces of other people's individuality, and had no sartorial existence of their own proper. Their walk was the walk of a race apart. They had a peculiar way of doggedly slinking round the corner, as if they were eternally going to the pawnbroker's. When they coughed, they coughed like people accustomed to be forgotten on doorsteps and in draughty passages, waiting for answers to letters in faded ink, which gave the recipients of those manuscripts great mental disturbance and no satisfaction. As they eyed the stranger in passing, they eyed him with borrowing eyes - hungry, sharp, speculative as to his softness if they were accredited to him, and the likelihood of his standing something handsome. (Little Dorrit I, ix; Dickens 1978: 91)

Auffällig für diesen Ausschnitt ist einerseits der Einsatz von Listen von Gegenständen, die gerade ob ihres Aufzählungscharakters keine wiedergabefähige Beschreibung liefern, sondern eine Atmosphäre evozieren. Andererseits sieht man hier auch Dickens' Einsatz von Metaphorik, die über eine Metonymie zwischen den Kleidungsstücken und dem Charakter der Beschriebenen funktioniert. So ist die Identität der Armen genauso zusammengeflickt wie ihre Kleidung; ihr Gang ist geprägt von ihrem häufigen Besuch des Pfandleihbüros, ihr Husten und ihr Blick ist determiniert von ihren erfolglosen Erfahrungen als Postboten und Bettler. Die Serie der Metonymien fügt sich zu einer großen Metapher, nach der die äußere Erscheinung der Armut das Wesen der Figuren bestimmt, sie also quasi die personifizierte Armut darstellen, die ihnen ein Aussehen oder ein Erscheinungsbild zuschreibt, das ihren Lumpen und ihrem schleichenden Fortbewegungsmodus ähnelt, sie quasi in diese verwandelt (Ähnlichkeit als Definiens der Metapher).

Beschreibungen sind besonders bei Dickens auch symbolisch und bedienen sich extensiver Metaphorik - sie zielen daher weniger darauf ab, einen genauen Grundriss zu liefern, sondern die Imagination des Lesers zu stimulieren und thematische Aspekte hervorzuheben. So ist die folgende Passage nicht darauf ausgerichtet, uns ein Inventar der angebotenen Gerichte zu liefern oder das Bistro so zu schildern, dass man die Räumlichkeiten detailgetreu nachzeichnen könnte, sondern die Köstlichkeit und Begehrtheit der Speisen drastisch darzustellen:

They walked on with him until they came to a dirty shop window in a dirty street, which was made almost opaque by the steam of hot meats, vegetables, and puddings. But glimpses were to be caught of a roast leg of pork bursting into tears of sage and onion in a metal reservoir full of gravy, of an unctuous piece of roast beef and blisterous Yorkshire pudding, bubbling hot in a similar receptacle, of a stuffed fillet of veal in rapid cut, of a ham in a perspiration with the pace it was going at, of a shallow tank of baked 
potatoes glued together by their own richness, of a truss or two of boiled greens, and other substantial delicacies. (Little Dorrit I, xx; Dickens 1978: 237)

Wenn im Roman Perspektive im Stanzelschen Sinn zu sehen ist, dann häufig im Zusammenhang einer gaze tour, d. h. aus der Beobachterperspektive. Der folgende Ausschnitt aus Hardys Under the Greenwood Tree ist hierfür ein gutes Beispiel:

Mr. Penny's was the last house in that part of the parish, and stood in a hollow by the roadside so that cart-wheels and horses' legs were about level with the sill of his shopwindow. This was low and wide, and was open from morning till evening, Mr. Penny himself being invariably seen working inside, like a framed portrait of a shoemaker by some modern Moroni. He sat facing the road, with a boot on his knees and the awl in his hand, only looking up for a moment as he stretched out his arms and bent forward at the pull, when his spectacles flashed in the passer's face with a shine of flat whiteness, and then returned again to the boot as usual. Rows of lasts, small and large, stout and slender, covered the wall which formed the background, in the extreme shadow of which a kind of dummy was seen sitting, in the shape of an apprentice with a string tied round his hair (probably to keep it out of his eyes). He smiled at remarks that floated in from without, but was never known to answer them in Mr. Penny's presence. Outside the window the upper-leather of a Wellington-boot was usually hung, pegged to a board as if to dry. (Under the Greenwood Tree II, i; Hardy 1998: 54)

Die Passage schildert, wie ein Passant Mr. Penny erlebt und gibt dem Leser ein eindrückliches Porträt. Trotzdem bleiben viele Aspekte dieser fiktionalen Szene offen, so dass ein Illustrator des Romans ganz verschiedene Visualisierungen inszenieren könnte. Insbesondere die Links-/Rechts-Platzierung des Schuhs bzw. auch des Gesellen bleiben offen.

Eine der wenigen Textbelege für eine perspektivische Raumbeschreibung vor dem 19. Jahrhundert ist in Sarah Scotts Millenium Hall (1762) zu finden:

The room where they [the women] sat was about forty-five feet long, of a proportionable breadth, with three windows on one side, which looked into a garden, and a large bow at the upper end. Over against the windows were three large book-cases, upon the top of the middle one stood an orrery, and a globe on each of the others. In the bow sat two ladies reading, with pen, ink, and paper on a table before them, at which was a young girl translating out of French. At the lower end of the room was a lady painting, with exquisite art indeed, a beautiful Madonna; near her another, drawing a landscape out of her own imagination; a third, carving a picture-frame in wood, in the finest manner; a fourth, engraving and a young girl reading aloud to them; the distance from the ladies in the bow-window being such, that they could receive no disturbance from her. At the next window were placed a group of girls [...]. (Millenium Hall; Scott 1995: 58-9)

Wieder handelt es sich um eine gaze tour des Besuchers, und wiederum ist die Deixis stark reduziert (upper erfordert einen Standort, der ,lower ${ }^{6}$ wäre). Hier allerdings könnte man eine grobe Zeichnung anfertigen.

Ich resümiere, dass der Vergleich des linguistischen Modells und auch von Stanzels Thesen mit den Romanpassagen einige Inkompatibilitäten aufweist.

(1) Die absolute Deixis der Vogelperspektive kommt offensichtlich nur in spezifischen (wissenschaftlichen) Textsorten und kaum in der Literatur vor. 
(2) Bei der literarischen Beschreibung von Innenräumen werden gaze und tour perspective verwendet, aber im Verhältnis zu den Textsorten der Linguisten sind deiktische Ausdrücke selten im Roman.

(3) Die Funktionen der fiktionalen Beschreibung sind offensichtlich ganz andere als diejenigen in realweltlichen Texten. So wollen faktuale Beschreibungen meist Anleitungen liefern, wie man sich zurechtfindet, während literarische Beschreibungen (auch wenn sie nicht metaphorisch sind) eher Atmosphäre evozieren, implizit (metonymische) Personen charakterisieren, symbolische Motive einführen oder die Imagination des Lesers im Sinne eines Barthes'schen effet de réel beflügeln (Barthes 1968). Die formalen Unterschiede sind daher funktionsbedingt.

Um diesen dritten Punkt noch weiter auszuführen, hier noch zwei Beispiele aus Gebrauchtsliteratur, die ihrerseits interessante Schlussfolgerungen erlauben:

Although now incorporating both nineteenth- and twentieth-century features and planting schemes, the extensive garden [of Montacute House, Somerset] still follows the outlines of the original layout. The oriel window at the north end of the long gallery looks down on a formal rectangle of trees and grass which lies on the site of the Elizabethan garden, the raised walks framing the sunken lawn with its bracelet of clipped yew and thorn and a nineteenth-century balustraded pond in the centre probably dating from when the house was built. A border of shrub roses under the retaining wall includes species in cultivation in the sixteenth century. The cedar lawn, with an arcaded garden house to which Lord Curzon added an Elizabethan façade, lies on the site of an old orchard. An avenue of mature cedars, beeches and limes, fronted by clipped Irish yew, frames the west drive created in 1851-52, its lines continued in the wide grassy ridge edged with limes which stretches away across the park to the east. (Houses of the National Trust; Greeves 2008: 218)

Turn left and walk along the river bank. The path is overgrown in places and the plank bridges can be slippery in wet weather but the views are superb. After $400 \mathrm{yds}(366 \mathrm{~m})$, climb the steps to your left to leave the river behind and turn left around a turf field. Keep to the edge of the field as it swings right and climbs between woodland to the left and a reservoir to the right. (Walks Through Britain's History; Phillips 2011: 67)

Während die zweite Passage eine klare route perspective verwendet und mit dem Pronomen you sowie deiktischem left und right operiert, ist die erste in ihrer Gestaltung den fiktionalen Beispielen viel verwandter. So fokussiert die Beschreibung von Montacute House auf dem salience-Prinzip: Was ist besonders bemerkenswert an diesem Landsitz? Die Informationsverteilung orientiert sich nicht an der Vermittlung grundlegender Grundriss-Aspekte. So wird die Existenz der long gallery vorausgesetzt und ihre Nord-Süd-Orientierung implizit durch „north end“ konstatiert. Je aufmerksamer man den Text studiert, desto verwirrter wird man, denn ein Nachzeichnen ist nicht möglich. Die Höhepunkte des Parks werden letztlich als eine Liste von sehenswerten Elementen präsentiert, deren Lokalisierung nur anhand einer Landkarte von Park und Haus möglich wird.

Dieser Befund ist nun insofern wieder interessant, weil er mit den Ergebnissen linguistischer Untersuchungen von Haus- und Wohnungsbeschreibungen korre- 
liert, in denen ein Nachzeichnen ebenfalls nicht funktioniert. So konstatieren Tversky et al., dass bei Wohnungsbeschreibungen nur ein sehr schneller Überblick gegeben wird und die Beschreiber z. B. nicht weitere Nebenräume diskursiv ,betreten', sondern nur das Zimmer, von dem aus diese Nebenräume wegführen. In einem Seminar zum Thema Beschreibung, das ich im Sommersemester 2014 gemeinsam mit Helga Kotthoff durchführte, hatten wir auch den Versuch gemacht, Wohnungsbeschreibungen erstellen zu lassen, die dann von anderen Seminarteilnehmern zeichnerisch realisiert werden sollten. Bei diesem Experiment stellte sich heraus, dass durchweg die Routenperspektive gewählt wurde, wodurch die Zeichner gerade jene Rahmeninformationen vermissten, die ihnen eine adäquate graphische Darstellung ermöglicht hätten. So erwähnte keine der Versuchspersonen, wie viele Räume die Wohnung insgesamt hatte oder wie der Grundriss angelegt wäre (also rechteckig, länglich oder L-förmig, etc.). Zudem wurde bei manchen Beschreibungen zwar der Versuch unternommen, Rauminhalte wiederzugeben, und die dabei gewählte Strategie lehnte sich an die gaze tour an; im Resultat war ein Nachzeichnen jedoch nur bedingt möglich, und die genaue Platzierung von Möbeln innerhalb des Wohnzimmers konnte nicht vermittelt werden. Andere Versuchspersonen griffen gleich auf eine aperspektivische Darstellung im Stanzelschen Sinn zurück und gaben nur eine Liste von Raumobjekten an. (Ullmer-Ehrich 1979: 69 hingegen zeigt, dass Raumbeschreibungen nach Blickachsen bzw. Rundumblicken strukturiert sind, also nicht notwendigerweise aperspektivisch sind.)

Es stellte sich auch heraus, dass viele Probleme beim Zeichnen auf unglückliche sprachliche Wendungen basierten, die zu Missverständnissen Anlass gaben. Ein Bespiel dafür ist der folgende Ausschnitt:
Also, du kommst in das Haus rein zur Haustür und stehst dann in einem Flur, der ist rechteckig, ähm, mehr lang als breit. Ähm, auf der linken Seite geht direkt die Treppe in den ersten Stock hoch und auf der rechten Seite kommt nach circa anderthalb Metern 'ne Tür, und von da aus kommt man in einen ganz großen Raum, der ist so lang wie das Haus. Ähm... dieser Raum ist Wohnzimmer, Esszimmer und Küche. Und zwar ist die Küche ganz vorne, also im Prinzip neben der Haustür - die Seite des Hauses. Ähm, dar- an schließt sich das Esszimmer an, und im hinteren Teil befindet sich dann das Wohn- zimmer. Ähm, diese Räume sind nicht durch Wände getrennt, sondern sind nur, ähm, sind... also dieser Raum ist komplett offen. Die einzige kleine Abtrennung, die es gibt, ist, dass zwischen Küche und Esszimmer so 'ne kleine Theke eingebaut ist. Ähm... im Esszimmer, also die Wand zwischen Esszimmer und Flur, da steht ein großer Kachel- ofen. Wenn man jetzt wieder im Flur ist, dann geht es links die Treppe rauf, und ne- ben der Treppe auf der linken Seite ist ein Gäste-WC. Neben dem Gäste-WC, also auch auf der linken Seite vom Flur, befindet sich ein Gästebadezimmer, und noch ein Stück weiter befindet sich ein Arbeitszimmer. Also der hintere Teil des Hauses besteht aus dem Stück Wohnzimmer und dem Arbeitszimmer; das bildet die kom- plette Breite des Hauses.

Hier versucht der Beschreiber, auf der Tour zu bleiben, verwirrt aber den Leser, so dass dieser erst beim mehrfachen Lesen der Transkription versteht, daß „links“ 
hier nicht in die Richtung der Eingangstür sondern auf der linken Seite des Ganges in Richtung des Flurs, auf der der Haustür entgegengewandten Seite, meint. Ullmer-Ehrich (1982) hat eine ähnliche Situation, wo jedoch der Interviewte Mißverständnisse durch explizites „auf der rechten Seite“ vorbeugt: „dann ist hier der Eingang [...] auf der linken Seite ist dann bei mir die Garderobe, das sind also drei Haken, und dann kommt der Kleiderschrank [...] wie halt so'n Kleiderschrank aussieht. Hm ja und dann geht's also direkt eh auf der rechten Seite diesmal in die Naßzelle rein" (243; Zitat bereinigt). Der Sprecher will sagen, daß von der Tür aus gesehen die Garderobe und der Schrank links sind, die Tür zur Nasszelle jedoch rechts ist, also nicht auf der linken Seite des Zimmers an den Schrank anschließt (rechts vom Schrank wenn man davorsteht). Da der Sprecher eine genaue Beschreibung des Kleiderschranks liefert, kommt er mit den hervorgehobenen Wörtern dann zurück zum Raumplan. Ullmer-Ehrich interpretiert dies als „explicit resumption“ und „a step back to some node in a branching structure" (1982: 244). ${ }^{6}$

Als Fazit dieses Vergleiches von faktualen und fiktionalen Beschreibungen ist also festzuhalten, dass selbst dort, wo Information weitergegeben werden soll, im mündlichen Beschreiben die Sprecher oft überfordert sind und die Perspektive des Zuhörers zu wenig im Blick haben. Dies ist sicher auch Übungssache, und der Versuch, die Beschreibung schriftlich zu fixieren, hilft, die Grundrisskomponente einzubringen. Trotz der deutlichen funktionalen Differenz zwischen fiktionalen und faktualen Beschreibungen ergibt sich überraschenderweise eine eher ähnliche Tendenz in der Performanz - Sprecher und zum Teil auch Schreiber haben nicht immer das Ziel und oft nicht das Geschick, Räumlichkeiten so zu schildern, dass man eine Zeichnung anfertigen könnte. Wie Ullmer-Ehrich (1979: 60) richtig bemerkt, dienen zwar Wegauskünfte der Orientierung bzw. haben „Anweisungscharakter“, aber „Wohnungs- und Wohnraumbeschreibungen“ wären „häufig“ dadurch gekennzeichnet, dass sie „Illustrationscharakter“ besäßen. Allerdings bleibt unklar, was genau die Funktion des Illustrierens wäre.

Dies führt mich zur letzten Frage: Warum hat man dennoch das Gefühl, eine Beschreibung sollte so genau sein, dass man die geschilderten Räume nicht nur visualisieren, sondern auch nachzeichnen können müsste? Darüber ließe sich lange spekulieren. Eine mögliche Erklärung wäre jedoch, dass wir seit dem 19. Jahrhundert in unserer Lebenswirklichkeit intensiv mit exakten Diagrammen und Nachzeichnungen konfrontiert sind. Nicht nur im well-made play wird in der RegieAnweisung genauestens vorgegeben, wo was zu stehen oder liegen oder hängen hat auf der Bühne; durch die Photographie und später den Film sowie die Verbreitung von Stadtplänen ist genaue Orientierung mit großer Präzision allgemein zugänglich geworden, ja zu einer Selbstverständlichkeit avanciert. Diese Präzision generiert Erwartungshaltungen, welche sich auf den Roman übertragen, und auch

6 Ullmer-Ehrich (1979: 73) kommentiert eine tatsächliche Fehlleistung, wo der Interviewte dann die Zuhörer in die Irre leitet. 
zu gegenläufigen Strategien im Theater (Beckett) und Roman (Josipovici) geführt haben - Literaten protestieren gegen die Erwartung, alles genauestens beschreiben und darstellen zu müssen. Die Enttäuschung über die Betonung wesentlicher Sinneseindrücke und den Luxus freier Imagination in literarischen Beschreibungen vor dem 19. Jahrhundert und darüber hinaus würde sich meiner Ansicht nach gut durch die Medienverwöhntheit des Publikums erklären lassen.

$\mathrm{Zu}$ welchen Schlussfolgerungen führt uns das bezüglich der Unterschiede von Fiktionalität und Faktualität? Literarische Beschreibung ist multifunktional und weitaus komplexer als faktuale Beschreibung. Literarische Beschreibung hat auch keinen Test zu bestehen - wenn Leser sich einen Schauplatz nicht so prägnant vorstellen können, ergeben sich keine ernsten Folgen. Ein schlecht geschriebener Reiseführer oder Wanderführer, der Leser in die Irre leitet, hat hingegen deutlich negative Auswirkungen. Auf der Straße ist man als Wegsuchender dem Zufall ausgeliefert, ob befragte Passanten einem zum Ziel verhelfen oder in die falsche Richtung weisen. Auch die Selektion der Informationen kann wichtige Informationen verschleiern - so hat ein Bekannter von mir eine Wohnung in Oxford für mich erkundet und das Fehlen eines Backofens nicht bemerkt. Auch in faktualen Beschreibungen kann man nicht von einer wirklichkeitsgetreuen Wiedergabe des Raumes ausgehen, und sogar Photos (wie man von den unliebsamen Überraschungen weiß, die man beim Bezug von Ferienwohnungen erlebt) sind nicht immer zuverlässige Deskriptoren des Realen. Aus diesem Grund haben, so meine ich, Linguisten und Literaturwissenschaftler in der Untersuchung von Beschreibungen noch viel voneinander zu lernen.

\section{Literatur}

Aristoteles (1994) Poetik: griechisch-deutsch. Hg. und Übers. Manfred Fuhrmann. Stuttgart: Reclam.

Bal, Mieke (1982) „On Meanings and Descriptions“. Studies in Twentieth Century Literature 6.1-2: 100-48.

Bareis, J. Alexander (2008) Fiktionales Erzäblen: Zur Theorie der literarischen Fiktion als Make-Believe. Göteborg: Acta Universitatis Gothoburgensis.

Barthes, Roland (1968) „L'Effet de Réel“. Communications 11: 84-89. Dt. Übersetzung: Barthes, Roland (2006) „Der Wirklichkeitseffekt“. Das Rauschen der Sprache: Kritische Essays IV. Übers. Dieter Hornig. Frankfurt a. M.: Suhrkamp. 164-72.

Bonheim, Helmut (1982) The Narrative Modes: Techniques of the Short Story. Cambridge: D.S. Brewer.

Brown, Julia Prewitt (2008) The Bourgeois Interior. Charlottesville, VA: Univ. of Virginia Press.

Bühler, Karl (1934) Sprachtheorie: Die Darstellungsfunktion der Sprache. Jena: G. Fischer. 
Chatman, Seymour (1978) Story and Discourse: Narrative Structure in Fiction and Film. Ithaca: Cornell Univ. Press.

- (1990) Coming to Terms: The Rhetoric of Narrative in Fiction and Film. Ithaca: Cornell Univ. Press.

Cohn, Dorrit (1999) The Distinction of Fiction. Baltimore: Johns Hopkins Univ. Press.

Defoe, Daniel (1986) A Tour Through the Whole Island of Great Britain [1724-26]. Hg. Pat Rogers. Harmondsworth: Penguin.

Dickens, Charles (1989) Hard Times [1854]. Hg. Paul Schlicke. Oxford: Oxford Univ. Press.

- (1978) Little Dorrit [1855-57]. Hg. Hablot Knight Browne. London: Oxford Univ. Press.

Fielding, Sarah (1994) The Adventures of David Simple [1744]. World's Classics. Hg. Malcolm Kelsall. Oxford: Oxford Univ. Press.

Fludernik, Monika (1993) The Fictions of Language and the Languages of Fiction: The Linguistic Representation of Speech and Consciousness. London: Routledge

- (1996) Towards a ,Natural Narratology. London: Routledge.

- (2000) „Genres, Text Types, or Discourse Modes - Narrative Modalities and Generic Categorization“. Style 34.2: 274-92.

- (2001) „Fiction vs Non-Fiction: Narratological Differentiations“. Erzäblen und Erzäbltheorie im 20. Jahrbundert. Festschrift für Wilhelm Füger. Hg. Jörg Helbig. Heidelberg: Winter. 85-103.

- (2005) „Unreliability vs. Discordance: Kritische Betrachtungen zum literaturwissenschaftlichen Konzept der erzählerischen ,Unzuverlässigkeit"“. ,Was stimmt denn jetzt?' Unzuverlässiges Erzäblen in Literatur und Film. edition text + kritik. Hgg. Fabienne Liptay und Yvonne Wolf. München: Boorberg. 39-59.

- (2013) Erzähltheorie: Eine Einfübrung. 4., verbesserte Auflage. Darmstadt: Wissenschaftliche Buchgesellschaft.

- (2014/15) „Description and Perspective: The Representation of Interiors“. Style 48.4. Im Druck.

Franzen, Johannes (2014) „Fiktionskritik der Gegenwart“. Vortrag auf der Tagung des GRK 1767 - Geschichte der Fiktionalität vom 8. Nov. 2014. Albert-LudwigsUniversität, Freiburg.

Genette, Gérard (1993) Fiction and Diction [Fiction et diction 1991]. Übers. Catherine Porter. Ithaca, NY: Cornell Univ. Press.

Greeves, Lydia (2008) Houses of the National Trust. London: National Trust.

Hamon, Philippe (1981) Introduction à l'analyse du descriptif. Paris: Hachette.

- (1982) „What is a description?" French Literary Theory Today. Hg. Tzvetan Todorov. Cambridge: Cambridge Univ. Press. 147-78.

Hardy, Thomas (1998) Under the Greenwood Tree [1872]. Hg. Tim Dolin. London: Penguin.

Hempfer, Klaus (1973) Gattungstheorie: Information und Synthese. München: Fink. 
- (1990) „Zu einigen Problemen einer Fiktionstheorie“. Zeitschrift für französische Sprache und Literatur 100: 109-37.

Hildesheimer, Wolfgang (1981) Marbot: eine Biographie. Frankfurt: Suhrkamp.

Hogan, Patrick Colm (2013) Narrative Discourse: Authors and Narrators in Literature, Film and Art. Columbus: Ohio State Univ. Press.

Jahn, Manfred (1999) „More Aspects of Focalization: Refinements and Applications“. In Pier: 85-110.

Klein, Christian, und Matías Martínez (2009) Hgg. Wirklichkeitserzäblungen: Felder, Formen und Funktionen nicht-literarischen Erzäblens. Stuttgart: Metzler.

Klein, Wolfgang (1979) „Wegauskünfte“. Zeitschrift für Literaturwissenschaft und Linguistik 9.33: 9-57.

Klotz, Peter (2013) Beschreiben: Grundzüge einer Deskriptologie. Berlin: Schmidt.

Konrad, Eva MAria (2014) „Panfiktionalismus“. Fiktionalität. Ein interdisziplinäres Handbuch. Hgg. Tobias Klauck und Tilmann Köppe. Berlin: de Gruyter. 23554.

Köppe, Tilmann, und Jan Stühring (2011) „Against Pan-Narrator Theories“. Journal of Literary Semantics 40.1: 59-80.

Köppe, Tilmann, und Tobias Klauk (2014) Hgg. Fiktionalität: ein interdisziplinäres Handbuch. Berlin: de Gruyter.

Köppe, Tilmann, Tobias Klauk und Dolf Rami (2014) Hgg. The Semantics of Fictional Discourse. Sonderausgabe des Journal of Literary Theory 8. Im Druck.

Kotthoff, Helga (2012): „Lehrer(inne)n und Eltern in Sprechstunden in Sprechstunden an Grund- und Förderschulen. Zeitschrift für Gesprächsforschung. Zur interaktionalen Soziolinguistik eines institutionellen Gesprächstyps“. Gesprächsforschung: Online-Zeitschrift für verbale Interaktion 13: 290-321.

Labov, William, und J. Waletzky (1968) „Narrative Analysis“. A Study of the NonStandard English of Negro and Puerto Rican Speakers in New York City. Hg. William Labov. New York: Columbia Univ. Press. 286-338.

Linde, Charlotte, und William Labov (1975) „Spatial Networks as a Site for the Study of Language and Thought“. Language 51.4: 924-39.

Lodge, David (1977) „Types of Description“. The Modes of Modern Writing: Metaphor, Metonymy and the Typology of Modern Literature. London: Arnold. 93-103.

Löschnigg, Martin (1999) „Narratological Categories and the (Non-)Distinction between Factual and Fictional Narratives". GRAAT 21: 31-48.

Marcus, Amit (2008) „We are You: The Plural and the Dual in ,We‘ Fictional Narratives". Journal of Literary Semantics 37: 1-21.

Margolin, Uri (1996) „Telling Our Story: On ,We“ Literary Narratives“. Language and Literature 5: 115-33.

- (2000) „Telling in the Plural: From Grammar to Ideology“. Poetics Today 21.3: 591-618. 
Melville, Herman (2008) „The Paradise of Bachelors and the Tartarus of Maids [1855]“. Melville's Short Fiction 1853-1856 [1977]. Hg. William B. Dillingham. Athens, Georgia: Univ. of Georgia Press. 183-207.

Mosher, Harold F. Jr. (1991) „Toward a Poetics of ,Descriptized“ Narration“. Poetics Today 12.3: 425-45.

Nünning, Ansgar (1989) Grundzüge eines kommunikationstheoretischen Modells der erzäblerischen Vermittlung: Die Funktion der Erzäblinstanz in den Romanen George Eliots. Horizonte 2. Trier: Wissenschaftlicher Verlag.

- (2007) „Towards a Typology, Poetics and History of Description in Fiction“. In Wolf/Bernhart: 91-128.

Pepys, Samuel (1997) The Concise Pepys. Hg. Stuart Sim. Wordsworth Classics. Ware: Herts.

Phillips, Charles (2011) Hg. Walks Through Britain's History. Basingstoke: AA Publishing.

Pier, John (1999) Hg. Recent Trends in Narratological Research: Papers from the Narratology Round Table, ESSE4, September 1997, Debrecen, Hungary and Other Contributions. GRAAT 21. Tours: Presses Univ. François Rabelais.

Richardson, Brian (2011) „U.S. Ethnic and Postcolonial Fiction: Toward a Poetics of Collective Narratives". Analyzing World Fiction: New Horizons in Narrative Theory. Hg. Frederick Luis Aldama. Austin: Univ. of Texas Press. 3-16.

Ryan, Marie-Laure (1997) „Postmodernism and the Doctrine of Panfictionality“. Narrative 5.2: 165-87.

- (2001) Narrative as Virtual Reality: Immersion and Interactivity in Literature and Electronic Media. Baltimore, MD: Johns Hopkins Univ. Press.

- (2004) Hg. Narrative Across Media: The Languages of Storytelling. Lincoln, Neb.: Univ. of Nebraska Press.

- (2005) „Panfictionality“. The Routledge Encyclopedia of Narrative Theory. Hgg. David Herman, Manfred Jahn und Marie-Laure Ryan. London: Routledge. 416-18.

Schama, Simon (1991) Dead Certainties. London: Granta.

Schmid, Wolf (2005) Elemente der Narratologie. Narratologia 8. Berlin: de Gruyter.

Schwittala, Johannes (2012) „Raumdarstellung in Alltagserzählungen“. Erzäblen als Form - Formen des Erzäblens. Germanistische Linguistik 295. Hgg. Friederike Kern, Miriam Morek und Sören Ohlhus. Berlin: de Gruyter. 161-200.

Scott, Sarah (1995) A Description of Millenium Hall [1762]. Hg. Gary Kelly. Peterborough, ONT: Broadview.

Sell, Roger (2000) Literature As Communication: The Foundations of Mediating Criticism. Pragmatics and beyond 78. Amsterdam: Benjamins.

Stanzel, Franz Karl (1984) A Theory of Narrative. Cambridge: Cambridge Univ. Press.

- (2008) Theorie des Erzäblens [1979]. 8. Aufl. Göttingen: Vandenhoek und Ruprecht. 
Steiner, Wendy (1995) The Scandal of Pleasure: Art in an Age of Fundamentalism. Chicago: Univ. of Chicago Press.

Sternberg, Meir (1974) „What is Exposition? An Essay in Temporal Delimitation". The Theory of the Novel: New Essays. Hg. John Halperin. New York: Oxford Univ. Press. 25-70.

- (1981) „Ordering the Unordered: Time, Space, and Descriptive Coherence“. Yale French Studies 61.2: 60-88.

Taylor, Holly A., und Barbara Tversky (1992) „Spatial Mental Models Derived from Survey and Route Descriptions". Journal of Memory and Language 31: 26192.

Taylor, Holly A., und Barbara Tversky (1996) „Perspective in Spatial Descriptions". Journal of Memory and Language 35: 371-91.

Trollope, Anthony (1990) Barchester Towers [1857]. World's Classics. Oxford: Oxford Univ. Press.

Tversky, Barbara, Paul Lee und Scott Mainwaring (1999). „Why Do Speakers Mix Perspectives?". Spatial Cognition and Computation 1: 399-412.

Ullmer-Ehrich, Veronika (1979) „Wohnraumbeschreibungen“. Zeitschrift für Literaturwissenschaft und Linguistik 9.33: 58-83.

- (1982) „The Structure of Living Space Descriptions“. Speech, Place, and Action. Studies in Deixis and Related Topics. Hgg. Robert J. Jarvella und Wolfgang Klein. Chichester: John Wiley \& Sons Ltd. 219-49.

Wall, Cynthia Sundberg (2006) The Prose of Things: Transformations of Description in the Eighteenth Century. Chicago: Univ. of Chicago Press.

Walton, Kendall (1990) Mimesis as Make-Believe: On the Foundations of Representational Arts. Cambridge, Mass.: Harvard Univ. Press.

Weinrich, Harald (1985) Tempus: Besprochene und erzäblte Welt [1964]. Sprache und Literatur 16. Stuttgart: Kohlhammer.

Wolf, Werner, und Walter Bernhart (2007) Hgg. Description in Literature and Other Media. Studies in Intermediality 2. Amsterdam: Rodopi.

Zipfel, Frank (2001) Fiktion, Fiktivität, Fiktionalität: Analysen zur Fiktion in der Literatur und zum Funktionsbegriff in der Literaturwissenschaft. Berlin: Erich Schmidt. 
